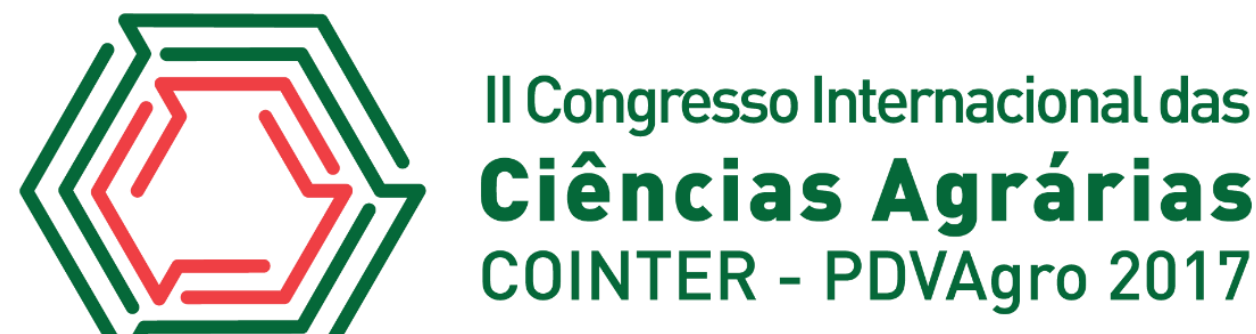

\section{QUALIDADE FÍSICA DO CAMARÃO (Litopenaeus vannamei) TRATADO COM PLASMA NÃO TÉRMICO}

\author{
Apresentação: Pôster \\ Julianna Paula do Vale Figueiredo ${ }^{1}$; Lucas Oliveira de Soares Rebouças ${ }^{2}$; Vanessa Clarice \\ Fernandes Alves ${ }^{3}$; Renata Bezerra Gomes ${ }^{4}$; Patrícia de Oliveira Lima ${ }^{5}$
}

\section{Introdução}

Após a captura, o pescado sofre uma série de alterações bioquímicas, físicas, químicas e biológicas, que tem início pela ação autolítica de enzimas musculares que hidrolisam proteínas e gorduras (GONÇALVES, 2006; HAARD, 2002). Em seguida, ocorre a ação dos microrganismos, provocando assim, alterações químicas e físicas no pescado, causando assim sua deterioração (HAARD, 2002). Fatores como qualidade, vida de prateleira e segurança dos alimentos são determinadas pela deterioração e o crescimento dos microrganismos patogênicos (McMEEKIN et al., 1993).

Por demonstrar eficácia no tratamento de alimentos, o plasma não térmico vem ganhando espaço, pois sua ação pode ser benéfica para os alimentos, prolongando a vida de prateleira de produtos frescos, mantendo assim a segurança alimentar (SMEU, 2014).

O objetivo do presente estudo foi avaliar o uso do plasma não térmico como uma alternativa para garantir a qualidade física do camarão branco (Litopenaeus vannamei) inteiro fresco.

\section{Fundamentação Teórica}

Apesar de ser um alimento de alto valor nutricional, o pescado pode oferecer risco ao consumidor (ALBERTINI, OETTERER, PRADO FILHO, 1997), pois, é o alimento de origem animal com maior probabilidade de deterioração por apresenta $\mathrm{pH}$ próximo a neutralidade, elevada

\footnotetext{
${ }^{1}$ Pós-graduação em Produção Animal, Universidade Federal Rural do Semi-árido, vanessaclaricefa@gmail.com

2 Pós-graduação em Ciência Animal, Universidade Federal Rural do Semi-árido, lucaslosr@gmail.com

${ }^{3}$ Pós-graduação em Ciência Animal, Universidade Federal Rural do Semi-árido, juliannafigueiredo@hotmail.com

${ }^{4}$ Pós-graduação em Ciência Animal, Universidade Federal Rural do Semi-árido, carlacampelo2@hotmail.com

${ }^{5}$ Professor(a) adjunto IV, Universidade Federal Rural do Semi-árido, pattlima@ufersa.edu.br
} 
atividade de água nos tecidos, alto teor de nutrientes facilmente utilizáveis pelos micro-organismos, além de acentuado teor de fosfolipídios e rápida ação destrutiva das enzimas presentes nos tecidos e nas vísceras do peixe (GASPAR, 1997; LEITÃO, 1997).

As alterações autolíticas, proteolíticas, oxidativas e microbiológicas vão desencadeando mudanças sensoriais e favorecem a deterioração do pescado (SANT’ANA; FREITAS, 2011). Outra consequência destas reações é o aparecimento de melanose em lagostas e camarões, fenômeno que ocasiona perda da qualidade visual do pescado pelo aparecimento de pontos pretos, embora não acarrete em alteração nutricional (FURLAN, 2011).

A aplicabilidade do plasma não térmico tem no tratamento antimicrobiano tem demostrado um futuro promissor na indústria alimentícia, pois, ao interagir com as células bacterianas inativam de forma eficaz uma gama de microrganismos, incluindo esporos e vírus (MISRA et al., 2011). Essa eficácia antimicrobiana do tratamento com plasma a frio pode ser benéfica para alimentos, a fim de prolongar a vida de prateleira de produtos frescos e manter a segurança ao longo de toda a cadeia alimentar (SMEU, 2014).

Esta tecnologia pode ser utilizada para a descontaminação superficial de produtos onde os microrganismos estão localizados externamente, podendo ser usada para desinfecção das superfícies antes da embalagem ou como parte integrante do processo de embalagem (BANU, 2012).

\section{Metodologia}

Foram utilizados aproximadamente $20 \mathrm{~kg}$ de camarão (L. vannamei) inteiro, fresco, pesando aproximadamente 10 gramas, sem uso de nenhum tipo de aditivo químico, proveniente de fazenda de cultivo localizada no nordeste do Brasil. Os camarões foram pesados e separados em quatro grupos amostrais, o grupo Controle (C) e os grupos onde as amostras foram expostas ao tratamento do plasma à frequências de $5 \mathrm{MHz}, 10 \mathrm{MHz}$ e $15 \mathrm{MHz}$, por 10 minutos. As amostras foram posicionadas dentro de uma placa de petri com dimensões de $80 \mathrm{~mm}$ de diâmetro e $15 \mathrm{~mm}$ de profundidade. O plasma permaneceu durante o tratamento a uma distância de aproximadamente 3 mm das amostras (não havendo contato efetivo entre o plasma e os camarões tratados, logo, não houve formação de descargas localizadas).

Para a determinação da qualidade física das amostras foram realizadas em triplicata, onde foram determinados o pH (AOAC 2005), cor avaliando a variação durante o armazenamento $(\Delta \mathrm{e}=$ 
$\left[\left(\mathrm{L}^{*}-\mathrm{L}^{*} 0\right)^{2}+\left(\mathrm{a}^{*}-\mathrm{a}^{*} 0\right)^{2}+\left(\mathrm{b}^{*}-\mathrm{b}^{*} 0\right)^{2}\right]^{1 / 2}$, ) utilizando as coordenadas $\mathrm{L}^{*}, \mathrm{a}^{*}$ e $\mathrm{b}^{*}$ (YUAN et al., 2016), capacidade de retenção de água - CRA (HAMM, 1960), perda de peso por cocção - PPC (WARRIS, 2003) e força de cisalhamento - FC (QUEIROGA et al., 2014) e posteriormente, os dados foram submetidos a análise de variância (ANOVA), e aos testes de comparação de média, teste t de Student e teste de Turkey, ao nível de 5\% de significância.

\section{Resultados e Discussões}

$\mathrm{O} \mathrm{pH}$ das amostras se mostrou estável até o $3^{\circ}$ dia de armazenamento, passando a variar a partir do $6^{\circ}$ dia. Houve um acréscimo no grupo controle, sendo significativamente superior às amostras tratadas. Já as amostras submetidas ao plasma mantiveram o pH estável, permanecendo dentro da legislação (RIISPOA, 1952) até o final do experimento. De acordo com López-Caballero et al., (2007) os crustáceos possuem altos índices de nitrogênio não-proteico, facilitando a atividade bacteriana ou de algumas enzimas endógenas, tais atividades resultam na elevação do pH durante o armazenamento.

Em relação a cor, foi observado um aumento gradativo nos valores de $\Delta \mathrm{e}$ com o aumento do tempo de armazenamento. A utilização do plasma foi eficaz na manutenção da cor dos camarões, sendo a frequência de $15 \mathrm{MHz}$ a mais eficiente dentre os tratamentos, e o grupo controle, obteve a maior variação de cor durante o armazenamento. Segundo Hsu et al., (2017) um dos principais parâmetros de qualidade para determinação do frescor do camarão é a cor, e o responsável variação da cor $(\Delta \mathrm{e})$ em crustáceos é o desenvolvimento da melanose, processo resultante da ação da enzima polifenoloxidase na presença de oxigênio. No presente estudo, a ocorrência do processo de melanização foi maior no grupo controle, explicando os maiores valores de $\Delta \mathrm{e}$. A utilização do plasma frio atmosférico, foi eficiente no retardo do processo de melanização, consequentemente resultando em menores variações de $\operatorname{cor}(\Delta \mathrm{e})$ no camarão durante o armazenamento.

O plasma contribuiu significativamente $(\mathrm{p}<0,05)$ para o aumento da capacidade de retenção de água (CRA) nas amostras, demonstrando uma relação proporcional na retenção de líquido. Para Wachirasiri et al., (2016) a CRA está diretamente associada ao pH da carne, amostras com pH de baixa acidez faz com que ocorra rápida desnaturação das proteínas, consequentemente diminuindo a capacidade da carne reter líquido. De acordo com Moreno, Loureiro e Souza (2008), a CRA influencia a aparência da amostra e determina a suculência no momento do consumo. 
Tabela 1: Caracterização física em função do tempo de armazenamento do Litopenaeus vannamei, submetido ou não à exposição do plasma não térmico.

\begin{tabular}{|c|c|c|c|c|c|}
\hline \multirow{2}{*}{$\begin{array}{l}\text { Days of } \\
\text { storage }\end{array}$} & \multirow{2}{*}{ Variáveis } & \multirow[b]{2}{*}{ Controle } & \multicolumn{3}{|c|}{ Aditivos } \\
\hline & & & $5 \mathrm{MHz}$ & $10 \mathrm{MHz}$ & $15 \mathrm{MHz}$ \\
\hline 0 & \multirow{6}{*}{$\mathrm{pH}$} & $6,1 \mathrm{Cf}$ & $6,2 \mathrm{Be}$ & $6,2 \mathrm{Bd}$ & $6,4 \mathrm{Ab}$ \\
\hline 1 & & 6,3 Ae & $6,3 \mathrm{Ad}$ & 6,3 Acd & $6,3 \mathrm{Ac}$ \\
\hline 3 & & $6,5 \mathrm{Bd}$ & $6,4 \mathrm{Bd}$ & $6,3 \mathrm{Bcd}$ & $6,4 \mathrm{Abc}$ \\
\hline 6 & & $6,7 \mathrm{Cc}$ & $6,5 \mathrm{Cc}$ & $6,3 \mathrm{Bc}$ & $6,3 \mathrm{Abc}$ \\
\hline 9 & & $6,8 \mathrm{Db}$ & $6,7 \mathrm{Cb}$ & $6,6 \mathrm{Bb}$ & $6,4 \mathrm{Abc}$ \\
\hline 12 & & 7,7 Ca & $6,9 \mathrm{Ba}$ & $6,9 \mathrm{Ba}$ & 6,7 Aa \\
\hline 0 & \multirow{6}{*}{$\Delta \mathrm{e}$} & $6,1 \mathrm{Cf}$ & $6,2 \mathrm{Be}$ & $6,2 \mathrm{Bd}$ & $6,4 \mathrm{Ab}$ \\
\hline 1 & & 6,3 Ae & $6,3 \mathrm{Ad}$ & $6,3 \mathrm{~cd}$ & $6,3 \mathrm{Ac}$ \\
\hline 3 & & $6,5 \mathrm{Bd}$ & $6,4 \mathrm{Bd}$ & $6,3 \mathrm{~cd}$ & $6,5 \mathrm{Abc}$ \\
\hline 6 & & $6,7 \mathrm{Cc}$ & $6,5 \mathrm{Cc}$ & $6,3 \mathrm{c}$ & $6,3 \mathrm{Abc}$ \\
\hline 9 & & $6,8 \mathrm{Db}$ & $6,7 \mathrm{Cb}$ & $6,6 \mathrm{~b}$ & $6,4 \mathrm{Abc}$ \\
\hline 12 & & 7,7 Ca & $6,9 \mathrm{Ba}$ & $6,9 \mathrm{a}$ & 6,7 Aa \\
\hline 0 & \multirow{6}{*}{ CRA $(\%)$} & $67,0 \mathrm{Ca}$ & $72,9 \mathrm{Ba}$ & $75,1 \mathrm{Ba}$ & $79,5 \mathrm{Aa}$ \\
\hline 1 & & $67,3 \mathrm{Da}$ & $70,8 \mathrm{Cab}$ & $75,2 \mathrm{Ba}$ & $80,3 \mathrm{Aa}$ \\
\hline 3 & & $67,0 \mathrm{Ca}$ & $68,3 \mathrm{BCbc}$ & $70,5 \mathrm{Bb}$ & $75,2 \mathrm{Ab}$ \\
\hline 6 & & $66,3 \mathrm{Ca}$ & 70,9 Bab & $71,7 \mathrm{Bb}$ & $74,7 \mathrm{Ab}$ \\
\hline 9 & & $64,1 \mathrm{Ca}$ & $67,2 \mathrm{Bc}$ & $69,7 \mathrm{Bb}$ & $72,9 \mathrm{Ab}$ \\
\hline 12 & & $59,8 \mathrm{Cb}$ & $68,5 \mathrm{Bbc}$ & $69,9 \mathrm{Bb}$ & $74,2 \mathrm{Ab}$ \\
\hline 0 & \multirow{6}{*}{$\mathrm{PPC}(\%)$} & $45,4 \mathrm{Ab}$ & $42,1 \mathrm{ABbc}$ & $39,4 \mathrm{BCc}$ & $37,0 \mathrm{Cb}$ \\
\hline 1 & & 46,6 Abc & $44,3 \mathrm{Abc}$ & $40,4 \mathrm{Bbc}$ & $39,2 \mathrm{Bab}$ \\
\hline 3 & & $46,7 \mathrm{Abc}$ & $43,4 \mathrm{ABbc}$ & $42,2 \mathrm{Bbc}$ & $40,3 \mathrm{Bab}$ \\
\hline 6 & & 46,9 Abc & $44,7 \mathrm{ABbc}$ & $41,7 \mathrm{Bbc}$ & $41,2 \mathrm{Ba}$ \\
\hline 9 & & $49,7 \mathrm{Ab}$ & 46,7 ABab & $44,1 \mathrm{BCab}$ & $41,8 \mathrm{Ca}$ \\
\hline 12 & & $54,1 \mathrm{Aa}$ & $48,9 \mathrm{Ba}$ & $46,2 \mathrm{BCa}$ & $42,7 \mathrm{Ca}$ \\
\hline 0 & \multirow{6}{*}{$\begin{array}{c}\text { Força de } \\
\text { Cisalhamento } \\
\left(\mathrm{kgf} / \mathrm{cm}^{2}\right)\end{array}$} & 1,1 Aa & 1,1 Aa & 1,2 Aab & $1,1 \mathrm{Aab}$ \\
\hline 1 & & $1,1 \mathrm{Aa}$ & 1,0 Aab & $1,2 \mathrm{Aa}$ & $1,2 \mathrm{Aa}$ \\
\hline 3 & & $0,8 \mathrm{Ab}$ & 1,0 Aab & $0,9 \mathrm{Ac}$ & 1,0 Aabc \\
\hline 6 & & $0,8 \mathrm{Ab}$ & 0,9 Aabc & $0,9 \mathrm{Abc}$ & $0,9 \mathrm{Abcd}$ \\
\hline 9 & & 1,0 Aab & $0,7 \mathrm{Bbc}$ & $0,9 \mathrm{Bbc}$ & $0,8 \mathrm{ABcd}$ \\
\hline 12 & & $0,7 \mathrm{Ab}$ & $0,8 \mathrm{Ac}$ & $0,8 \mathrm{Ac}$ & $0,6 \mathrm{Ad}$ \\
\hline
\end{tabular}

A,B Letras maiúsculas distintas na linha indicam diferença entre os tratamentos pelo teste Tukey 5\%. ${ }^{\mathrm{a}, \mathrm{b}, \mathrm{c}}$ Letras minúsculas distintas na coluna indicam diferença entre os tempos de armazenamento pelo teste Tukey 5\% 
Segundo Freire et al., (2016), por meio do processo de cocção, várias características do produto são alteradas, dentre elas os teores de gordura e umidade, consequentemente, interferindo no peso/volume do alimento, sendo uma característica importante para o rendimento final. As menores perdas de peso ocorreram nas amostras tratadas com plasma, sendo o grupo tratado com a frequência de $15 \mathrm{MHz}$, o mais satisfatório, pois mostrou-se mais eficiente quando comparado aos demais grupos. Apresentando uma relação inversamente proporcional com a CRA.

Em relação à força de cisalhamento, não houve diferença entre as amostras tratadas com plasma e ao grupo controle, os valores variaram de 0,6 a $1,2 \mathrm{kgf} / \mathrm{cm}^{2}$. Com o tempo de armazenamento, a força de cisalhamento das amostras foi diminuindo, atingindo os valores mais baixos ao final do experimento.

\section{Conclusões}

As amostras tratadas com plasma não térmico obtiveram resultados mais satisfatórios, sendo o tratamento sob frequência de $15 \mathrm{MHz}$ o mais eficiente para as características físicas do camarão.

\section{Referências}

ALBERTINI, S.; OETTERER, M.; PRADO FILHO, L. G. Fontes de contaminação e toxicologia por chumbo. Boletim da Sociedade Brasileira de Ciência e Tecnologia de Alimentos, Campinas, 1997; 31 (2): 137-47.

AOAC. Official methods of analysis of the Association Analytical Chemists. 18.ed. Gaithersburg, Maryland, 2005.

BANU, M. S. Cold Plasma as a Novel Food Processing Technology. International Journal of Emerging Trends in Engineering and Developments, 2012.

BARBOSA NUNES QUEIROGA, Inês Maria et al. Qualidade sensorial do camarão Litopenaeus vannamei congelado. Semina: Ciências Agrárias, v. 35, n. 4, 2014.

BRASIL. Ministério da Agricultura, Pecuária e Abastecimento. Decreto no 30.691, de 29 de março de 1952. Aprova o novo Regulamento de Inspeção Industrial e Sanitária de Produtos de Origem Animal (RIISPOA). Diário Oficial [da] União. Brasília, DF, 7 jul. 1952. Seção 1, p. 10785.

FREIRE, Bárbara Camila Firmino et al. Qualidade de camarão (Litopenaeus vannamei) minimamente processado. Acta Veterinaria Brasilica, v. 10, n. 2, p. 150-155, 2016.

GASPAR, J.;VIEIRA, R.;TAPIA, M. Aspectos Sanitários do pescado de origem de água doce e marinha , comercializado n afeira de Gentilândia, Fortaleza, Ceará. Revista Ciência e Tecnologia de Alimentos, São Paulo. v.11, p.20-287,1997. 
GONÇALVES, A. A. Ocorrência de off-flavor em pescado: um problema a ser resolvido em peixes marinhos. Revista aquicultura \& pesca; 2006; 18:30-1.

GOÑI, S.M.; SALVADORI, V.O. Prediction of cooking times and weight losses during meat roasting. Journal of Food Engineering, Essex, v.100, p.1-11, 2010.

HAARD N. The role of enzymes in determining seafood color, flavor and texture. In: Bremmer HA (ed.). Safety and quality issues in filsh processing. Cambridge (England): Woodhead Publishing Limited and CRC Press LLc; 2002. 520 p.

HAMM, R. Biochemistry of meat hydratation: advances in food research. Cleveland. v. 10, p.335$443,1960$.

HSU, Francis LK (Ed.). Kinship and culture. Routledge, 2017.

LEITÃO, M. F., RIOS, D. P., GUIMARÃES, J. G., BALDINI, V. L. S., \& MAINARDES PINTO, C. S. Alterações químicas e microbiológicas em pacu (Piaractus mesopotamicus) armazenado sob refrigeração a $5^{\circ}$ C. Food Science and Technology (Campinas), 1997. v. 17, p. 160-166, 1997.

LÓPEZ-CABALLERO, Maria Elvira et al. Quality of thawed deepwater pink shrimp (Parapenaeus longirostris) treated with melanosis-inhibiting formulations during chilled storage. International journal of food science \& technology, v. 42, n. 9, p. 1029-1038, 2007.

McMEEKIN, T., OLLEY, J., ROSS, T., RATKOWSKY, D., 1993. Basic concepts and methods. In Predictive microbiology: theory and application. Research Studies Press Ltd, Taunton, UK, pp. $11-84$.

MORENO, G. M. B; LOUREIRO, C. M. B.; SOUZA, H. B. A. Características qualitativas da carne ovina. Revista Nacional da Carne, São Paulo, n.381, p.76-90, 2008.

MUCHENJE, V. et al. Some biochemical aspects pertaining to beef eating quality and consumer health: A review. Food Chemistry, v. 112, n. 2, p. 279-289, 2009.

SANT'ANA, L. S.; FREITAS, M. Q. Aspectos sensoriais do pescado. GONÇALVES, AA Tecnologia do pescado: ciência, tecnologia, inovação e legislação. São Paulo: Atheneu, 2011.AOAC. Official methods of analysis of the Association Analytical Chemists. 18.ed. Gaithersburg, Maryland, 2005.

SMEU, I.; IOANANICOLAU, A. Enhancement of food safety - Antimicrobial effectiveness of cold plasma treatments. The Annals of the University Dunarea de Jos of Galati Fascicle VI Food Technology, v. 38, n. 1, p. 9-20, 2014.

WACHIRASIRI, Kulraphat et al. Use of amino acids as a phosphate alternative and their effects on quality of frozen white shrimps (Penaeus vanamei). LWT-Food Science and Technology, v. 69, p. 303-311, 2016.

WARRIS, P. D. Ciência de la Carne. Acribia: Zaragoza, 2003. 309p.

YUAN, Gaofeng et al. Effect of chitosan coating combined with pomegranate peel extract on the quality of Pacific white shrimp during iced storage. Food control, v. 59, p. 818-823, 2016. 